\title{
Erratum to: A new concept and a comprehensive evaluation of SYSMEX UF-1000i flow cytometer to identify culture-negative urine specimens in patients with UTI
}

\author{
T. Monsen ${ }^{1} \cdot$ P. Ryden ${ }^{2,3}$
}

Published online: 15 July 2017

(C) Springer-Verlag GmbH Germany 2017

\section{Erratum to: Eur J Clin Microbiol Infect Dis DOI 10.1007/s10096-017-2964-1}

The original version of this article unfortunately contains an error in the abstract. The corrected abstract is published below.

\begin{abstract}
Urinary tract infections (UTIs) are among the most common bacterial infections in men and urine culture is gold standard for diagnosis. Considering the high prevalence of culture-negative specimens, any method that identifies such specimens is of interest. The aim was to evaluate a new screening concept for flow cytometry analysis (FCA). The outcomes were evaluated against urine culture, uropathogen species and three conventional screening methods. A prospective, consecutive study examined 1,312 urine specimens, collected during January and February 2012. The specimens were analyzed using the Sysmex UF1000i FCA. Based on the FCA data culture negative specimens were identified in a new model by use of linear discriminant analysis (FCA-LDA). In total 1,312
\end{abstract}

The online version of the original article can be found at http://dx.doi. org/10.1007/s10096-017-2964-1

T. Monsen

tor.monsen@ climi.umu.se

1 Department of Clinical Microbiology, Umeå University Hospital, and University of Umeå, SE-90185 Umeå, Sweden

2 Department of Mathematics and Mathematical Statistics, Umeå University, Umeå, Sweden

3 Computational Life science Cluster (CLiC), Umeå University, Umeå, Sweden patients were included. In- and outpatients represented $19.6 \%$ and $79.4 \%$, respectively; $68.3 \%$ of the specimens originated from women. Of the 610 culture-positive specimens, Escherichia coli represented 64\%, enterococci $8 \%$ and Klebsiella spp. 7\%. Screening with FCA-LDA at 95\% sensitivity identified $42 \%(552 / 1312)$ as culture negative specimens when UTI was defined according to European guidelines. The proposed screening method was either superior or similar in comparison to the three conventional screening methods. In conclusion, the proposed/suggested and new FCA-LDA screening method was superior or similar to three conventional screening methods. We recommend the proposed screening method to be used in clinic to exclude culture negative specimens, to reduce workload, costs and the turnaround time. In addition, the FCA data may add information that enhance handling and support diagnosis of patients with suspected UTI pending urine culture.

The original article has been corrected. 